\title{
Obesidade, Desnutrição e Pobreza: a Insegurança Alimentar e Nutricional na Otica do Espaço Social Alimentar
}

\section{Obesity, Undernutrition and Poverty: Food Insecurity and Nutritional in the Social Food Space}

\author{
Cícero Augusto Silveira Braga
}

Lorena Vieira Costa ${ }^{a}$

\begin{abstract}
Resumo: O presente trabalho busca analisar a insegurança alimentar e nutricional (IAN) através de uma abordagem objetiva. Entendendo a multidimensionalidade do tema, o trabalho o mensura considerando três aspectos: ingestão calórica insuficiente, obesidade e proporção da renda gasta com alimentação. Essas medidas são escolhidas a fim de se entender as diferentes dimensões da IAN, aliadas às mudanças de hábito alimentar da população brasileira e aos diferentes impactos no bem-estar domiciliar. Utiliza-se, para tal, os dados da Pesquisa de Orçamento Familiar (2008). Os resultados são estimados a partir de três distintas regressões logísticas. As evidências mostram que as características do ambiente e do domicílio estão especialmente ligadas à IAN quando medida pela vulnerabilidade de renda, evidenciando a provisão de serviços públicos de qualidade, além da estrutura urbana/rural, como fatores que impactam na IAN. Conclui-se que o tema possui um caráter multidimensional e, portanto, aponta-se a necessidade de uma análise a partir de suas particularidades, exigindo esforços para uma metodologia mais complexa.
\end{abstract}

Palavras-chave: Insegurança alimentar. Obesidade. Ingestão calórica. Análise de vulnerabilidade.

Abstract: The present work seeks to analyze food and nutritional insecurity (FNI) through an objective approach. Understanding the theme's multidimensionality, the work measure it through insufficient caloric intake, obesity and proportional expenditure of income with food. These measures were chosen in order to understand FNI dimensions, due to the changes in the Brazilian population's eating habits and, the different impacts on the household welfare. The data used is from POF (2008) and the results were estimated from three different logistic regressions. The results showed that the characteristics of the environment and the household are especially related to FNI when measured by income vulnerability, showing that the provision of quality public services as well as urban/rural structure are factors that impact on FNI. In addition, the correlation analysis of the variables evidences the need to advance the measurement of the theme, in order to encompass its particularity and multidimensionality. It reinforces, above all, the multidimensional character of the theme, pointing out the need to analyze the theme based on its particularities and demanding efforts for a more complex methodology.

a Universidade Federal de Viçosa (UFV), Programa de Pós Graduação em Economia Aplicada (PPGEA), Departamento de Economia Rural (DER). Viçosa, Minas Gerais, Brasil. 
Keywords: Food insecurity. Obesity. Energy intake. Vulnerability.

JEL Classification: I310; I150; J100.

\section{Introdução}

O tema da alimentação e da insegurança alimentar e nutricional (IAN) em suas diversas dimensões está diretamente relacionado ao desenvolvimento econômico. Isso se dá uma vez que não só a alimentação é um elemento que garante a soberania da população, mas também porque está diretamente ligada à produtividade do trabalho e a um ciclo de pobreza (BANERJEE; DUFLO, 2011).

As mais recentes e diversas agendas para o desenvolvimento internacional e nacional vêm pautando a necessidade de se entender a segurança alimentar como imperativo para que se enxergue o desenvolvimento de uma nação. É o caso, por exemplo, dos objetivos do milênio propostos pela Organização das Nações Unidas (ONU), que exprime como primeiro objetivo erradicar a extrema pobreza e a fome, reduzindo pela metade a proporção de pessoas famintas (UNITED NATIONS, 2015a). Mais recentemente, a agenda 2030 para o desenvolvimento sustentável também manifesta como objetivo central acabar com a fome no mundo e alcançar um estado de segurança alimentar, enfatizando a necessidade de se erradicar todas as formas de má nutrição (UNITED NATIONS, 2015b).

Normalmente, a IAN é estudada a partir de três dimensões, especificamente: o acesso, que trata dos atributos econômicos e físicos da capacidade individual de acessar os alimentos, como questões logísticas, de renda e preços; a disponibilidade, que abarca as etapas produtivas e de comercialização, bem como políticas alimentares e agrárias; e a suficiência, que trata dos aspectos de utilização e qualidade alimentar, relacionando questões fisiológicas e sanitárias, entendendo as condições de transformar os alimentos em fontes vitais necessárias (ONU, 2017).

Essas três dimensões estão intimamente ligadas especialmente no que diz respeito à região em que se analisa. Nesse sentido, o espaço social, além de ser determinante quando se analisam variáveis macroeconômicas (como escoamento e acesso ao alimento, por exemplo), deve ser considerado também ao se perceber as particularidades que rodeiam o domicílio ou a unidade de análise. É o que sugere a teoria do espaço social alimentar (POULAIN; PROENÇA, 2003), que elucida a importância da análise do espaço social e sua relação com a alimentação, de maneira geral.

Os trabalhos empíricos que estudam o tema a nível domiciliar para o Brasil utilizam especialmente os dados provenientes da Escala Brasileira de Insegurança Alimentar (Ebia), sejam aqueles aplicados juntamente à Pesquisa Nacional por Amostra de Domicílios (PNAD) ou quando aplicada em pesquisa de campo 
(CAMELO; TAVARES; SAIANI, 2009; OLIVEIRA et al., 2010; SEGALL-CORRÊA; MARIN-LEON, 2009). Entretanto, mesmo sendo a medida mais robusta ${ }^{1}$ e utilizada na literatura, existe uma crítica em relação à adequação dessa medida dita subjetiva, uma vez que parte da percepção individual e não consegue captar a multidimensionalidade do tema (HOFFMANN, 2008). Dessa forma, fazem-se necessárias medidas que consigam captar objetivamente vulnerabilidades e deficiências da população no que tange à IAN.

São limitados, portanto, os estudos que consigam sobrepujar o entendimento padrão da IAN e analisem distintas perspectivas do tema, buscando identificar relações específicas que reforçam o estado de vulnerabilidade dos domicílios brasileiros; especificamente, que captem os efeitos da transição nutricional vivenciada em países em desenvolvimento, em que são percebidas, ao mesmo tempo, domicílios com moradores em situação de obesidade e desnutrição (COUTINHO; GENTIL; TORAL, 2008).

Nesse sentido, dadas as limitações encontradas na literatura e a mudança demográfica vivenciada no Brasil, o presente estudo busca relacionar como as diferentes perspectivas da IAN são observadas nas diferentes configurações domiciliares brasileiras. Para isso, a IAN é tratada a partir de três diferentes medidas objetivas: desnutrição, captada pela ingestão insuficiente de calorias; vulnerabilidade econômica (alto gasto proporcional da renda domiciliar com alimentação); e obesidade, calculada pelos valores do índice de massa corpórea (IMC). A escolha dessas três medidas se dá no intuito de captar as diferentes dimensões e perceber, a partir de diferentes óticas, o impacto da insegurança alimentar, exercício central para o entendimento do tema, como apontam Cafiero et al. (2014) e Kepple e Segall-Corrêa (2011).

Busca-se identificar como as características relacionadas ao indivíduo, ao domicílio e ao ambiente intensificam a probabilidade de o domicílio enfrentar IAN. Foram utilizados os dados da Pesquisa de Orçamentos Familiares (POF) 2008-2009 (IBGE, [2009]), ${ }^{2}$ estimados em um modelo de regressão logística que fornece as razões de chances do acontecimento da IAN.

Essa proposta, além de estender o entendimento da IAN, traz à tona novas particularidades da população vivenciadas através das mudanças de hábitos alimentares que estão relacionadas com o bem-estar. Além disso, entender o ambiente alimentar permite que sejam identificadas relações entre a IAN e a realidade dos domicílios brasileiros, possibilitando propostas efetivas de políticas públicas mais efetivas de combate à IAN.

\footnotetext{
1 A Ebia nasce de uma extensa pesquisa iniciada no Departamento de Agricultura dos Estados Unidos e adaptada por pesquisadores para a realidade brasileira (SEGALL-CORREA; MARIN-LEON, 2009).

2 Apesar de defasada, esta é a pesquisa mais recente que contempla hábitos de consumo.
} 
O trabalho divide-se em mais quatro seções, além desta introdução: na Seção 2, apresenta-se conceitos e evidências empíricas sobre a insegurança alimentar e nutricional e o ambiente domiciliar. Na Seção 3, métodos, estratégia empírica e dados utilizados na pesquisa. Posteriormente, na Seção 4, discute-se os resultados e, por fim, na Seção5, as conclusões.

\section{A Insegurança Alimentar e Nutricional no Brasil e o Ambiente Domiciliar}

No Brasil, a segurança alimentar é entendida pela legislação como "[...] a realização do direito de todos ao acesso regular e permanente a alimentos de qualidade, em quantidade suficiente e sem comprometer o acesso a outras necessidades importantes tendo como base práticas alimentares promotoras de saúde [...]" (BRASIL, 2006). Em outras palavras, uma pessoa estará em situação de IAN a partir do momento em que lhe é restrito o acesso à alimentação balanceada e ingestão de nutrientes suficientemente para a manutenção de sua saúde, seja essa restrição integral ou em detrimento de outras necessidades.

No Brasil, as principais metodologias para a mensuração da IAN se diferenciam primeira e essencialmente pela base de dados. Pode-se destacar, por um lado, a análise subjetiva, que possui como referência principalmente a Ebia. Essa escala utiliza de análises psicométricas para medir o fenômeno da IAN. Entretanto, tal análise pode ser insuficiente uma vez que parte da percepção e experiência individual das pessoas entrevistadas, carecendo, portanto, de análises objetivas que consigam retratar os mais diversos elementos e dimensões da IAN (HOFFMANN, 2008).

Por outro lado, as análises objetivas utilizam principalmente os dados de renda e orçamento familiar, como aqueles provenientes da Pesquisa de Orçamento Familiar (POF). Por essa ótica, a questão da segurança alimentar pode ser medida a partir de nível de consumo calórico e nutricional e também por medidas antropométricas, por exemplo. Entretanto, limitam-se por se tratarem de medidas isoladas e, como no caso anterior, não abarcar a completude da IAN.

Essa divisão quanto à mensuração dificulta captar as especificidades da IAN. Por exemplo, as mudanças de hábitos alimentares, aliadas às heterogeneidades espaciais encontradas no Brasil, podem ser um ponto chave para que se entenda a situação da população que ainda vive sob a situação de IAN. Essa discussão é minuciosamente discutida por Poulain e Proença (2003), que buscam discutir a alimentação a partir das ciências sociais e indicam que, para isso, deve-se, em um primeiro momento, analisar as mudanças epistemológicas para, então, passar-se para a análise do espaço onde se inserem os indivíduos.

Dessa forma, na medida em que a população altera seus padrões de vida e hábitos alimentares, surgem novas preocupações no âmbito da segurança alimentar, como é o caso do excesso de peso e obesidade, que são fatores de risco para 
uma série de doenças como diabetes, disfunções cardiovasculares, cânceres, entre outras. No caso brasileiro, a Associação Brasileira para o Estudo da Obesidade e da Síndrome Metabólica (Abeso) mostrou que aproximadamente $60 \%$ da população brasileira está ao menos em situação de sobrepeso, número que subiu mais de 10\% em 10 anos (ABESO, 2015).

De acordo com a Organização das Nações Unidas para a Alimentação e a Agricultura (FAO; OPS, 2016), a coexistência de fome, desnutrição, sobrepeso e outras causas da insegurança alimentar nos países em desenvolvimento estão intimamente ligadas à falta de acesso a uma alimentação de qualidade que seja saudável e consiga fornecer os nutrientes necessários para a reprodução de uma vida ativa e saudável. Essa relação de acesso ao alimento, por sua vez, relaciona-se diretamente com a proporção da renda destinada à alimentação pela família. Quando esta é alta, encontra-se ainda mais vulnerável na presença de qualquer choque econômico, como o desemprego ou, até mesmo, mudanças na estrutura familiar (COSTA et al., 2015)por meio de um modelo de escolha binária (logit, justificando-se, portanto, o uso de tais medidas para o fornecimento de um novo panorama do tema.

Alguns trabalhos empíricos levam em consideração variáveis relacionadas às características do entorno do domicílio para explicarem a IAN vivenciada. Por exemplo, Alves e Boog (2007) apontam que, ao se tornarem provedores de seus próprios alimentos, estudantes tendem a mudar seu comportamento alimentar, uma vez que a qualidade, padrões de comensalidade e representações alimentares fornecem insumos para o desenvolvimento e promoção da saúde.

Costa et al. (2013) destacam variáveis estaduais e macrorregionais como determinantes nas relações, especialmente por estarem relacionadas com a produtividade da terra e a disponibilidade de alimentos. Já Costa et al. (2015)por meio de um modelo de escolha binária (logit propõem essa relação de maneira mais direta, destacando recursos do domicilio, como a presença de geladeira, água canalizada e rede de esgoto, como um bloco de controle de variáveis de infraestrutura. Em relação ao ambiente, é apontada a relação direta entre saneamento básico, tipo de habitação, presença de pessoas com verminoses, construção da moradia, dentre outras variáveis do entorno domiciliar e a IAN (PANIGASSI et al., 2008; PIMENTEL; SICHIERI; SALLES-COSTA, 2009).

Bezerra, Olinda e Pedraza (2017) percebem que existe maior prevalência de IAN em escolas e creches, serviços de saúde e entre beneficiários do Programa Bolsa Família, cujas variáveis como renda familiar, quantidade de indivíduos do domicílio e tipo de moradia mostraram-se como principais determinantes de IAN, evidenciando sua determinação social.

Tratando-se das regiões rurais, Yuyama et al. (2007) apontam que o fato de estas possuírem maior diversidade de alimentos é crucial para que as zonas rurais 
estejam menos sensíveis à IA. Isso implica que a população da região analisada consegue sobreviver com maior dignidade no que tange à alimentação, dada a oportunidade de plantio de diversificada gama de produtos alimentícios. Essa afirmação deve ser cuidadosa, porém, dadas as heterogeneidades encontradas no campo.

\section{Métodos}

Esta seção se dedica em apresentar os procedimentos metodológicos utilizados no trabalho. Para isso, na Seção 3.1, discute-se a medida de insegurança alimentar adotada. Posteriormente, na Seção 3.2, apresenta-se a o modelo empírico e o método logístico utilizado para estimar os resultados, bem como os dados e as variáveis utilizados.

\subsection{Identificação da Insegurança Alimentar e Nutricional}

No intuito de não só fornecer medidas objetivas, mas também abarcar o caráter multidimensional do tema, três medidas são consideradas para caracterizar a IAN. Essas medidas, de natureza objetiva (consideram o consumo alimentar reportado e as compara com valores limítrofes determinados internacionalmente), são proxies que permitem uma análise por diferentes perspectivas do mesmo fenômeno.

A primeira delas é o consumo insuficiente de calorias diárias. Segue-se a recomendação da Agência Nacional de Vigilância Sanitária - Anvisa (2001) de que cada indivíduo deve consumir, no mínimo, $80 \%$ da quantidade de calorias necessárias por dia, que são 2000 kcal. Esse número é referência para a quantidade mínima de calorias sugerida por ingestão diária que garante, ao mínimo, as necessidades básicas alimentares para fonte de energia. Esse valor varia de acordo com características individuais dos habitantes dos domicílios, principalmente entre crianças e adultos. Por isso, utiliza-se a escala de equivalência de habitantes do domicílio (ZAIDI; HAGENAARS; DE VOS, 1994), adaptada da originalmente proposta pela Organização para a Cooperação e Desenvolvimento Econômico (OCDE). Essa escala atribui valor igual a um para a pessoa de referência do domicílio, 0.5 para cada adulto adicional e 0.3 para cada criança, de modo que cada domicílio é representado pelo adulto equivalente $(\mathrm{AE}),{ }^{3}$ como mostra a equação 1 .

$$
A E=1+0.5\left(N_{\text {adultos }}-1\right)+0.3\left(N_{\text {crianças }}\right)
$$

A segunda medida para a IAN é a situação de obesidade da pessoa de referência do domicílio. Para a observação da obesidade, utiliza-se a medida antropo-

3 Como forma de ilustração, um domicílio que seja formado por três pessoas, sendo duas adultas e uma criança, terá como número equivalente 1,8 pessoas adultas. 
métrica de referência, o IMC. O seu cálculo é realizado de acordo com o indicado pela Organização Mundial da Saúde - OMS (WHO, 1995), através da razão entre o peso e o quadrado da altura. Segundo essa medida, os indivíduos podem ser considerados como "abaixo do peso", "intervalo normal", "acima do peso" e em situação de obesidade. Serão considerados como inseguros aqueles domicílios chefiados por pessoas obesas.

Por fim, a terceira medida de IAN baseia-se no gasto proporcional do domicílio com a alimentação. Essa medida é proposta por Smith (2002), que aponta que aqueles domicílios que destinam mais de $70 \%$ da sua renda para gastos com alimentação estão em situação de vulnerabilidade, uma vez que reflete em seu acesso a alimentos no futuro. Em outras palavras, ao destinar a maior parte de sua renda para a alimentação, os domicílios sofrerão limitações frente à possíveis choques econômicos, como o desemprego, por exemplo.

Em síntese, os valores limítrofes para cada variável dependente que determinará a situação de IAN estão definidos na Tabela 1.

Tabela 1 - Valores determinados para medir a IAN

\begin{tabular}{lll}
\hline Variável & Quantidade & Unidade de medida \\
\hline Calorias $^{1}$ & $<2000 \mathrm{kcal}$ & $\mathrm{Kcal} / \mathrm{dia} / \mathrm{AE}$ \\
$\mathrm{IMC}^{2}$ & $\geq 30$ & $\mathrm{IMC}\left(\mathrm{kg} / \mathrm{m}^{2}\right)$ \\
$\begin{array}{l}\text { Gastos com } \\
\text { alimentação }\end{array}$ & $\geq 70 \%$ & $\begin{array}{l}\text { Proporção de gastos da renda total domiciliar } \\
\text { com alimentação }(\mathrm{R} \$)\end{array}$ \\
\hline
\end{tabular}

Fonte: Elaboração própria.

\subsection{Estratégia Empírica}

Definida a situação de IAN a partir de três características qualitativas (presenciar ou não IAN), para captar os efeitos das características do espaço social alimentar utiliza-se um modelo logit (WOOLDRIDGE, 2007), que se baseia em uma função de distribuição logística acumulada. O modelo logístico apresenta as possibilidades de ocorrência do evento (equação 2), no qual a variável dependente $Y_{i j}$ está em função das informações dos indivíduos $(I)$, dos domicílios $(D)$ e do ambiente domiciliar $(A)$, todos seguidos de seus respectivos parâmetros, mais o termo constante:

$$
p\left(y_{i}=1 \mid D, I, A\right)=F\left(\beta_{0}+\beta_{1} D_{i}+\beta_{2} A_{i}\right)
$$


em que $Y_{i}$ é a variável dependente e diz respeito à situação de IAN, e i corresponde à ingestão calórica insuficiente, situação de obesidade da pessoa de referência e gastos superiores a 70\% da renda com alimentação.

Cada uma das variáveis explicativas assume valores um ou zero, dado que:

$Y_{i j}=1$, caso o j-ésimo domicílio presencie IAN com respeito à $i$;

$Y_{i j}=0$, caso contrário.

A diferença desse modelo para as regressões convencionais - geralmente feitas por mínimos quadrados ordinários (MQO) - é que a interpretação é baseada na probabilidade de que um evento aconteça, e não na estimação de valores médios, devido ao caráter dos dados. Entretanto, é útil para determinar uma relação direta ou indireta entre as variáveis.

A base de dados utilizada neste trabalho é a POF dos anos de 2008 e 2009 (IBGE, [2009]), e as variáveis foram ajustadas de acordo com seu peso amostral. Dessa pesquisa, foram considerados principalmente quatro registros: domicílio, pessoas, condições de vida e consumo alimentar. Extraído o nível de consumo domiciliar, utiliza-se, para a conversão das quantidades consumidas em calorias e gramas, a Tabela de Composição Nutricional dos Alimentos Consumidos no Brasil. Especificamente, utilizam-se as informações de energia na composição de alimentos por 100 gramas de parte comestível.

Da base de dados, são excluídas as informações duplicadas por domicílio, domicílios chefiados por pessoas menores de 15 anos e as que não possuem informações para a renda, escolaridade ou características referentes ao domicílio a fim de que as observações sejam mais realísticas e robustas. As variáveis explicativas consideradas serão aquelas relacionadas ao domicílio, ao indivíduo e ao ambiente em que se inserem os domicílios, listadas e descritas no Quadro 1. Foram utilizadas, ao todo, 32.630 observações. 


\section{Quadro 1 - Descrição das variáveis explicativas do modelo}

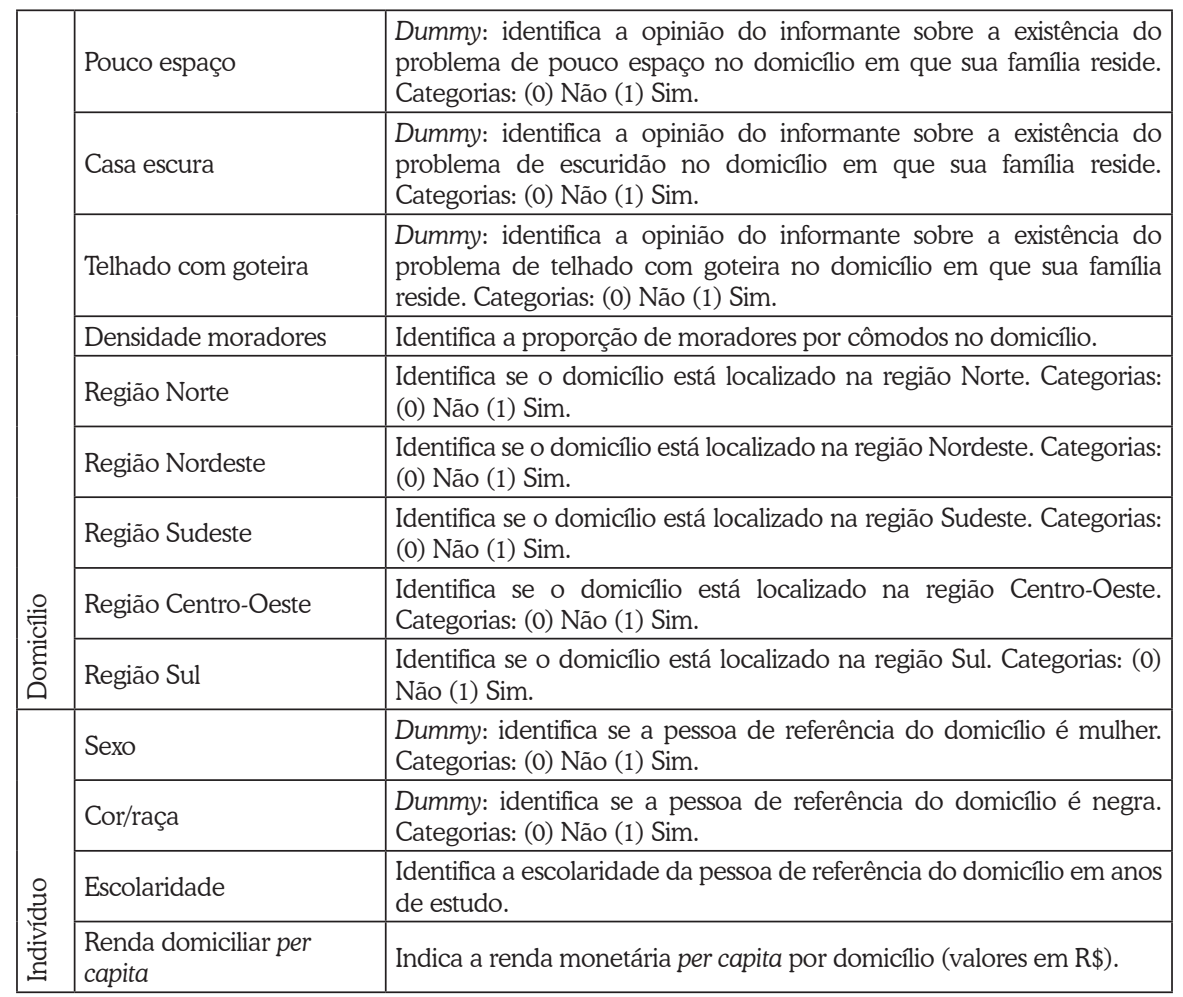

Continua... 
Conclusão.

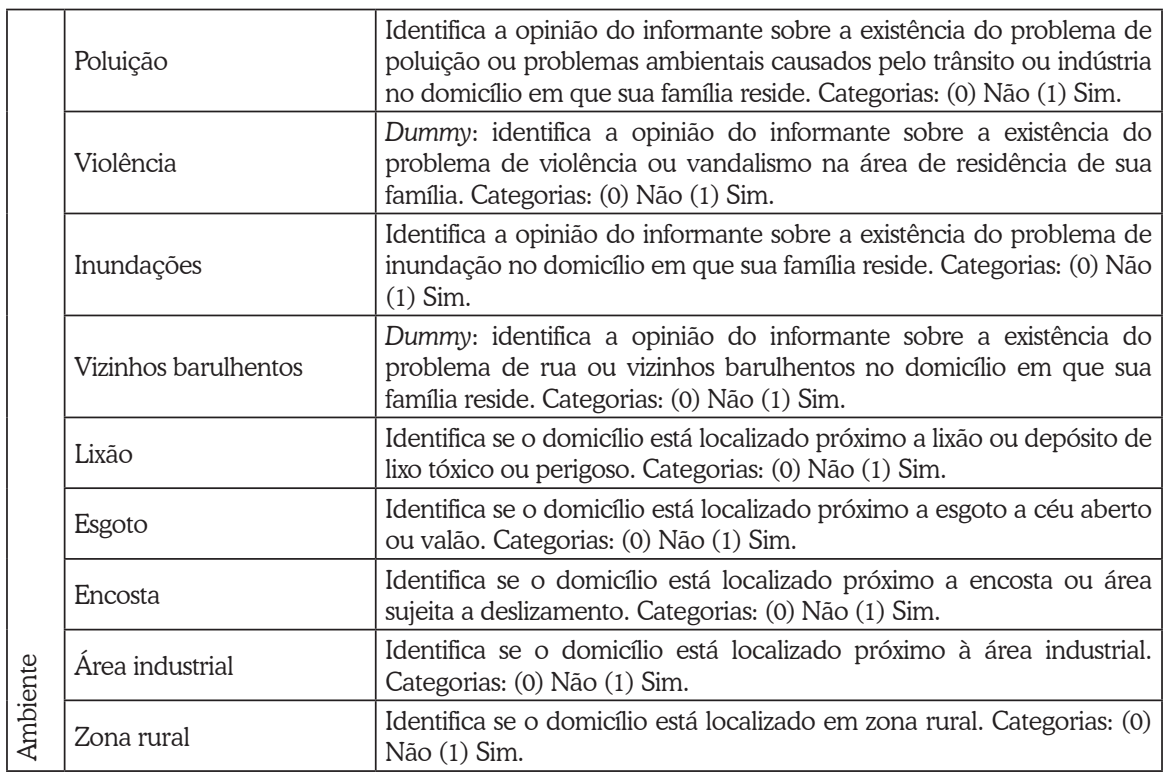

Fonte: Elaboração própria a partir de resultados da Pesquisa de Orçamento Familiar (IBGE, [2009]).

\section{Resultados e Discussões}

A Tabela 2 apresenta a matriz de correlações entre as definições de IAN conforme as variáveis escolhidas - calorias, obesidade e renda - e descreve a análise simultânea da associação entre as variáveis.

Tabela 2 - Matriz de correlação entre as variáveis de IAN

\begin{tabular}{llll}
\hline & Calorias & Obesidade & Renda \\
\hline Calorias & 1 & - & - \\
Obesidade & -0.0058 & 1 & - \\
Gastos & 0.0315 & -0.0338 & 1 \\
\hline
\end{tabular}

Fonte: Elaboração própria a partir dos dados da POF 2008-2009 (IBGE, [2009]).

Observa-se a quase inexistência de correlação entre elas ou, inclusive, correlação negativa, o que reforça o caráter multidimensional da IAN. Esses indicadores, ainda que isoladamente, conseguem captar diferentes dimensões da multidimensionalidade do tema, desafio apontado como essencial para o entendimento da IAN segundo (CARLETTO; ZEZZA; BANERJEE, 2013). Essa relação é esperada, ao passo 
que, cada uma das variáveis, abarca uma vertente específica da determinação da IAN. Por exemplo, o consumo inferior ao desejável de calorias sugere que o indivíduo não estará em situação de obesidade, uma vez que não acessa nem ao menos o mínimo calórico sugerido pela Anvisa. Além disso, sua inexpressiva correlação pode ser explicada uma vez que a ingestão calórica não está relacionada com a qualidade dietética daquele domicílio, tampouco considera outros gastos com alimentação, como os realizados fora do domicilio, por exemplo.

Dessa forma, comprova-se que a IAN é um fenômeno que pode - e deve - ser analisado por diferentes perspectivas, bem como propõe os modelos estimados, de acordo com o Quadro 2.

Quadro 2 - Razões de chance e coeficientes dos estimadores de IAN dos domicílios brasileiros (2008-2009)

\begin{tabular}{|c|c|c|c|c|}
\hline \multicolumn{2}{|c|}{ Variáveis } & Calorias & Obesidade & Renda \\
\hline \multirow{8}{*}{$\begin{array}{l}\text { Características do } \\
\text { indivíduo (chefe do } \\
\text { domicílio) }\end{array}$} & \multirow{2}{*}{ Sexo $^{1}$} & 1.5579 & 1.4224 & 1.2018 \\
\hline & & $(0.1166)^{* * *}$ & $(0.0352)^{* * *}$ & $(0.1933)^{* * *}$ \\
\hline & \multirow{2}{*}{ Cor/raça ${ }^{2}$} & 1.0330 & 0.9711 & 1.1675 \\
\hline & & $(0.0827)^{\mathrm{ns}}$ & $(0.0249)^{\mathrm{ns}}$ & $(0.0322)^{* * *}$ \\
\hline & \multirow{2}{*}{ Escolaridade } & 0.9855 & 0.9959 & 0.9667 \\
\hline & & $(0.0066)^{*}$ & $(0.0016)^{*}$ & $(0.0026)^{* * * *}$ \\
\hline & \multirow{2}{*}{$\begin{array}{l}\text { Renda domiciliar } \\
\text { per capita }\end{array}$} & 0.9999 & 1.0001 & . \\
\hline & & $(0.0001)^{*}$ & $(0.0000)^{* * *}$ & . \\
\hline \multirow{12}{*}{$\begin{array}{l}\text { Características do } \\
\text { domicilio }\end{array}$} & \multirow{2}{*}{ Pouco espaço } & 1.0902 & 0.9122 & 1.3794 \\
\hline & & $(0.0828)^{\mathrm{ns}}$ & $(0.02436)^{* * *}$ & $(0.0375)^{* * * *}$ \\
\hline & \multirow{2}{*}{ Casa escura } & 1.2690 & 0.9785 & 1.2807 \\
\hline & & $(0.1103)^{* * *}$ & $(0.0306)$ & $(0.0368)^{* * * *}$ \\
\hline & \multirow{2}{*}{$\begin{array}{l}\text { Telhado com } \\
\text { goteira }\end{array}$} & 0.8465 & 1.0561 & 0.7329 \\
\hline & & $(0.0922)^{*}$ & $(0.0278)^{* *}$ & $(0.1887)^{* * *}$ \\
\hline & \multirow{2}{*}{$\begin{array}{l}\text { Densidade } \\
\text { moradores }\end{array}$} & 0.9735 & 1.0074 & 0.8858 \\
\hline & & $(0.0299)^{\mathrm{ns}}$ & $(0.0087)^{\mathrm{ns}}$ & $(0.0096)^{* * * *}$ \\
\hline & \multirow{2}{*}{ Inundações } & 1.1649 & 1.0157 & 0.8555 \\
\hline & & $(0.1067)^{\mathrm{ns}}$ & $(0.0410)^{\mathrm{ns}}$ & $(0.3265)^{* * * *}$ \\
\hline & \multirow{2}{*}{$\begin{array}{l}\text { Vizinhos } \\
\text { barulhentos }\end{array}$} & 1.0088 & 1.0236 & 0.9276 \\
\hline & & $(0.0881)^{\mathrm{ns}}$ & $(0.0290)^{\mathrm{ns}}$ & $(0.0276)^{* * *}$ \\
\hline
\end{tabular}

Continua... 
Conclusão.

\begin{tabular}{|c|c|c|c|c|}
\hline \multicolumn{2}{|c|}{ Variáveis } & Calorias & Obesidade & Renda \\
\hline \multirow{22}{*}{$\begin{array}{l}\text { Características do } \\
\text { ambiente }\end{array}$} & \multirow{2}{*}{ Poluição } & 1.1812 & 1.0968 & 1.0578 \\
\hline & & $(0.1137)^{*}$ & $(0.0344)^{* *}$ & $(0.0363)^{*}$ \\
\hline & \multirow{2}{*}{ Violência } & 0.9702 & 1.0968 & 1.0626 \\
\hline & & $(0.0808)^{\mathrm{ns}}$ & $(0.0266)^{n s}$ & $(0.2999)^{* * *}$ \\
\hline & \multirow{2}{*}{ Lixão } & 0.7393 & 0.9938 & 1.1769 \\
\hline & & $(0.1701)^{\mathrm{ns}}$ & $(0.0687)^{\mathrm{ns}}$ & $(0.0772)^{* * *}$ \\
\hline & \multirow{2}{*}{ Esgoto } & 1.2474 & 1.0664 & 1.0239 \\
\hline & & $(0.1501)^{*}$ & $(0.0448)^{\mathrm{ns}}$ & $(0.0426)$ \\
\hline & \multirow{2}{*}{ Encosta } & 0.9202 & 0.9910 & 1.1042 \\
\hline & & $(0.1927)^{\mathrm{ns}}$ & $(0.0697)^{\mathrm{ns}}$ & $(0.0733)$ \\
\hline & \multirow{2}{*}{ Área industrial } & 1.0218 & 1.2205 & 0.7752 \\
\hline & & $(0.1716)^{\mathrm{ns}}$ & $(0.0622)^{* * *}$ & $(0.0485)^{* * * *}$ \\
\hline & \multirow{2}{*}{ Zona rural } & 0.8213 & 0.7765 & 1.4368 \\
\hline & & $(0.0788)^{*}$ & $(0.0248)^{* * * *}$ & $(0.0421)^{* * * *}$ \\
\hline & \multirow{2}{*}{ Região Norte } & 0.6220 & 0.8774 & 1.3005 \\
\hline & & $(0.0803)^{* * * *}$ & $(0.0394)^{* * *}$ & $(0.0620)^{* * * *}$ \\
\hline & \multirow{2}{*}{ Região Nordeste } & 0.7761 & 0.8556 & 1.9328 \\
\hline & & $(0.0826)^{*}$ & $(0.0315)^{* * *}$ & $(0.0782)^{* * * *}$ \\
\hline & \multirow{2}{*}{ Região Sudeste } & 0.8011 & 0.9297 & 0.9186 \\
\hline & & $(0.0916)^{*}$ & $(0.0354)^{*}$ & $(0.0427)^{*}$ \\
\hline & \multirow{2}{*}{ Região Sul } & 0.5841 & 1.1256 & 0.8027 \\
\hline & & $(0.0916)^{* * * *}$ & $(0.0505)^{*}$ & $(0.0475)^{* * * *}$ \\
\hline & LR chi (2) & $131.97 * * *$ & $601.60 * * *$ & $3281.96 * * *$ \\
\hline & Count $\mathrm{R}^{2}$ & 98.7 & 85.4 & 85.6 \\
\hline
\end{tabular}

Fonte: Elaboração própria a partir de dados da pesquisa.

Notas: Erros padrões entre parênteses; nível de significância: * $p<0.10$, ${ }^{* *} p<0.05$, ${ }^{* * *} p<$ 0.01; não significativa (ns); ${ }^{1}$ indica se a pessoa de referência é do sexo feminino; ${ }^{2}$ indica se a pessoa de referência do domicílio é autodeclarada preta ou parda.

No Quadro 2, encontram-se os resultados da regressão logística, que correspondem às razões de chance de que o domicílio (ou o chefe de família, no caso da obesidade) esteja em situação de IAN. Esse valor, quando maior que um, indica que a variável possui um impacto positivo sobre a variável dependente que representa a IAN, ou seja, aumenta a probabilidade do domicílio estar em situação de IAN; quando for menor que um, indica que o impacto daquele coeficiente é negativo, ou seja, reduz a probabilidade do domicílio encontrar-se em situação de IAN.

Todas as especificações, para todas as variáveis consideradas, tiveram um valor de razão de verossimilhança (likelihood radio - LR) com probabilidade menor que 0.000 , mostrando que, em seu conjunto, são estatisticamente diferentes de zero a 1\% de significância, indicando serem boas estimativas para explicação do modelo. Além disso, analisando-se o valor do count $R^{2}$, as especificações econométricas encontram-se bem ajustadas e conseguem prever pelo menos $85 \%$ das 
observações corretamente. Ainda, a distribuição mostrou-se homocedástica pelo teste de Breusch-Pagan.

Das variáveis determinantes da IAN, a que melhor apresentou resposta para mensuração do fenômeno foi a participação da renda nos gastos com a alimentação. Nesta, todas as variáveis selecionadas para a análise mostraram-se significativas, com exceção das especificidades do domicílio situar-se próximo ao esgoto e encosta. Essas variáveis também não apresentaram significância para nenhuma das outras duas variáveis dependentes postas na análise.

A primeira análise pode ser feita a partir das variáveis referentes à pessoa de referência do domicílio, determinantes para apontar a alocação dos recursos. As estimativas mostram, em consenso, que os domicílios chefiados por mulheres possuem mais chances de estarem em situação de IAN, enquanto aumentos em anos de estudo da pessoa de referência reduzem a probabilidade de estarem inseguros. Esses dados estão de acordo com o que é apresentado na bibliografia em relação aos dados empíricos. Essa observação vai ao encontro do que sugere a "feminização da pobreza", que discute as razões dos domicílios chefiados por mulheres serem aqueles a apresentarem maiores graus de vulnerabilidade. Segundo essa teoria, a mulher na posição de chefe de domicílio se vê incumbida não só do sustento da casa, como também das atividades do cuidado do lar. Por isso, precisam assumir diferentes turnos e modalidades de trabalho (como o trabalho doméstico não remunerado), fazendo com que o domicílio aumente a probabilidade de estar em situação de vulnerabilidade.

Por outro lado, aumentos em anos de estudo da pessoa de referência do domicílio sugere melhor habilidade para alocação dos recursos, além de maiores rendimentos médios. Dessa forma, a postura alimentar bem como a proporção da renda gasta com alimentos tendem a ser melhor vistas em domicílios onde o(a) chefe é mais instruído(a).

A variável correspondente à raça da pessoa de referência mostrou-se significativa somente para explicar a IAN pela ótica da renda: observa-se que domicílios chefiados por pessoas pretas e pardas têm mais chances de gastarem mais de 70\% de sua renda com alimentação. Esse resultado também é congruente se observado o perfil desses domicílios de forma geral, já que são aqueles com menor presença de eletrodomésticos e acesso a serviços, por exemplo. Além disso, são os (as) negros (as) que recebem, em média, os menores salários no Brasil.

Quanto à renda domiciliar per capita, apresentou-se neutra para determinação da IAN a partir das variáveis selecionadas, visto que o valor do seu coeficiente foi próximo à unidade. Entretanto, os efeitos da renda são melhores analisados quando postos pela variável dependente (como dado pela terceira especificação), já que esta consegue captar melhor as determinações da IAN. 
Partindo-se para a análise das características do domicílio em si, as estimativas mostraram-se contrárias ao esperado e também ao que sugere o modelo teórico. O que se observou é que se, por um lado, as características relacionadas ao pouco espaço e à casa escura representam maior probabilidade de o domicílio estar em situação de IAN para a análise a partir de renda e ingestão calórica, por outro elas possuem impacto oposto para a análise a partir da obesidade. As demais variáveis foram significativas para explicar o evento apenas a partir da análise da renda, apesar de irem de encontro com o que sugere a teoria do espaço social alimentar. Ainda, a presença de possíveis fatores negativos para um ambiente adequado, bem como o aumento da densidade de moradores, inundações e presença de vizinhos barulhentos reduzem as possibilidades de o domicílio sofrer IAN, contrariamente do que se sugere o modelo teórico e as evidências empíricas.

Especialmente no que diz respeito em relação ao consumo insuficiente de calorias e obesidade, as variáveis correspondentes ao domicílio traduzem o paradoxal cenário nutricional observado em diferentes países desenvolvidos. Segundo o relatório da OMS, com a mudança nos hábitos alimentares, cada vez mais apresentam-se, sobretudo em domicílios carentes, moradores obesos e desnutridos (WHO, 2017).

O resultado apresentado por tais variáveis reflete, mais uma vez, o caráter multidimensional do tema. Uma vez que a IAN pode ser analisada por diferentes óticas, é esperado que os determinantes para essa situação não sejam os mesmos. Dessa forma, é natural que as variáveis relacionadas à estrutura domiciliar sejam condicionantes, portanto, especialmente no que tange à IAN pela ótica da renda.

Passando, portanto, para as características do ambiente em que se insere o domicílio, mostrou-se, de forma robusta, que o fato de se situar em região com poluição leva-o a estar em IAN. É um resultado comum para as três especificações e reforça a necessidade de avanço de políticas ambientais, uma vez que a IAN está diretamente ligada ao bem-estar dos indivíduos e a seus hábitos alimentares. Além disso, dá-se destaque à relação dos gastos com o ambiente violento e a presença de lixão, na qual ambos apresentaram relação positiva com a situação de IAN.

Outras variáveis relacionadas ao ambiente dizem respeito à localização dos domicílios em áreas rurais. Tem-se que os domicílios rurais estão mais propensos a gastarem parte significativa de sua renda com a alimentação. Entretanto, são nesses domicílios que as probabilidades de não ingestão mínima calórica e presença de obesidade é menos provável. Ou seja, são mais vulneráveis se analisados pela renda, mas menos vulneráveis em relação à postura alimentar. Esse resultado está ligado às evidências de que produtores e moradores de áreas rurais percebem a IAN a partir de aspectos como qualidade dos alimentos, privação do consumo pela renda, fome e relações de troca, por exemplo. Além disso, é apontada a preferência dos domicílios rurais por alimentos naturais a industrializados, além da 
necessidade de acesso contínuo à educação para alimentação ou, ainda, o processamento de alimentos com "amor e cuidado".

Analisando-se pelas macrorregiões, ${ }^{4}$ os municípios das regiões Norte e Nordeste são mais propensos a terem seus gastos majoritariamente destinados à alimentação, estando, portanto, vulneráveis por essa ótica; Já a análise para as regiões Sudeste e Sul são contrárias, ou seja, há relação negativa com IAN. Por outro lado, analisando-se pelas variáveis relacionadas à obesidade e ingestão calóricas, há uma relação negativa entre as regiões e IAN.

\section{Considerações Finais}

Neste trabalho, a IAN é entendida como um processo complexo e multidimensional. Tais características, no entanto, dificultam a mensuração objetiva do fenômeno. Assim, três diferentes dimensões da IAN foram consideradas: aquela que deriva do consumo insuficiente de calorias, antropométricas (IMC) e de vulnerabilidade ao acesso futuro (gastos da renda com alimentação). Com essas definições, objetivou-se avaliar os fatores associados a cada caso com vistas a destacar possíveis aspectos comuns ou importantes disparidades quanto aos seus determinantes. Em particular, buscou-se identificar os efeitos dos ambientes interno e externo e das características pessoais das famílias brasileiras para maior ou menor propensão à IAN.

Apesar do modelo proposto contemplar diferentes abordagens do tema, não permite a análise em nível da IAN, apenas das probabilidades de ocorrência (através do modelo logit). As variáveis, de maneira geral, mostraram-se mais bem comportadas para uma análise do tema a partir dos gastos com renda, uma vez que foi para essa especificação que a maioria apresentou-se significativa. A análise do modelo do espaço social alimentar falha se analisado a partir da ingestão calórica e de medidas antropométricas, indicando que não necessariamente o espaço social alimentar está ligado à transição nutricional, mostrando-se um problema estrutural da nova dinâmica social.

Entre as variáveis explicativas analisadas, as evidências trazidas em relação ao gênero, à renda, à densidade de moradores por cômodo e à moradia na zona rural mostraram-se as mais relevantes. Apesar de as variáveis não terem sido significativas, de maneira geral, em relação às características do entorno do domicílio, destacaram-se, principalmente, aquelas que dizem respeito à proximidade a lixão e esgoto. Isso mostra alguma inconsistência, uma vez que manifestaram-se mais vulneráveis (na perspectiva da IAN) aqueles domicílios que estão próximos a esses espaços, mesmo que, em média, sua avaliação tenha sido positiva.

4 A análise por macrorregiões leva em consideração a região Centro-Oeste como parâmetro. 
Finalmente, vê-se que o ambiente em que se insere o domicílio está mais diretamente ligado à sua vulnerabilidade econômica no que diz respeito à alimentação. Posto isso, prova-se que a provisão de serviços públicos de qualidade, aliada à garantia de direitos mínimos, como saneamento básico e tratamento de lixo, por exemplo, pode ser um fator que ajude a erradicar a IAN dos domicílios brasileiros.

Ademais, dadas as particularidades encontradas na base de dados, sugere-se, para pesquisas futuras, um recorte especial apenas para os domicílios que apresentem vulnerabilidades específicas como estarem perto de áreas degradantes como o lixão ou esgoto, por exemplo. Uma vez que a relação acesso, disponibilidade e suficiência - que são sugeridas por variáveis macrorregionais e individuais mostraram-se críveis para a análise, tal recorte pode sustentar melhor as relações existentes nos casos de IAN. Além disso, urge a necessidade para determinação objetiva que seja consistente e consiga abarcar as diferentes variáveis que possam impactar a situação de IAN, seja a nível macro ou microeconômico.

\section{Referências}

ABESO. Quase 60\% dos brasileiros estão acima do peso, revela IBGE. São Paulo, 2015. Disponível em: https://abeso.org.br/quase-60-dos-brasileiros-estao-acima-do-peso-revela-ibge/. Acesso em: 10 dez. 2020.

ALVES, H. J.; BOOG, M. C. F. Comportamento alimentar em moradia estudantil: um espaço para promoção da saúde. Revista de Saúde Pública, v. 41, n. 2, p. 197-204, 2007.

ANVISA. Resolução - RDC n 40, de 21 de março de 2001. Brasilia, 2001.

BANERJEE, A. V.; DUFLO, E. Poor economics: a radical rethinking of the way to fight global poverty. 1. ed. New York: PublicAffairs, 2011.

BEZERRA, T. A.; OLINDA, R. A.; PEDRAZA, D. F. Insegurança alimentar no Brasil segundo diferentes cenários sociodemográficos. Ciência ES Saúde Coletiva, v. 22, n. 2, p. 637-651, 2017.

BRASIL. Lei n. ${ }^{\circ} 11.346$, de 15 de setembro de 2006. Cria o Sistema Nacional de Segurança Alimentar e Nutricional - SISAN com vistas em assegurar o direito humano à alimentação adequada e dá outras providências. Brasília, 2006. Disponível em: <http://www.planalto. gov.br/ccivil_03/_Ato2004-2006/2006/Lei/L11346.htm>. Acesso em: 20 set. 2011.

CAFIERO, C. et al. Validity and reliability of food security measures. New York Academy of Sciences, v. 1331, n. 1, p. 230-248, 2014.

CAMELO, R. S.; TAVARES, P. A.; SAIANI, C. C. S. Alimentação, nutrição e saúde em programas de transferência de renda: evidências para o programa bolsa família. EconimiA, v. 10, n. 4, p. 685-713, 2009. 
CARLETTO, C.; ZEZZA, A.; BANERJEE, R. Towards better measurement of household food security: harmonizing indicators and the role of household surveys. Global Food Security, v. 2, n. 1, p. 30-40, 2013.

COSTA, L. V. et al. Produtividade agrícola e segurança alimentar dos domicílios das regiões metropolitanas brasileiras. Revista de Economia e Sociologia Rural, v. 51, n. 4, p. 661-680, 2013.

COSTA, L. V. et al. Fatores associados à segurança alimentar nos domicílios brasileiros em 2009. Economia e Sociedade, v. 23, n. 2, p. 373-394, 2015.

COUTINHO, J. G.; GENTIL, P. C.; TORAL, N. A desnutrição e obesidade no Brasil: o enfrentamento com base na agenda única da nutrição. Cadernos de Saúde Pública, v. 24, n. 2, p. 332-340, 2008.

FAO; OPS. América Latina y el Caribe: panorama de la seguridad alimentaria y nutricional. 1. ed. Santiago: FAO, 2016.

HOFFMANN, R. Determinantes da insegurança alimentar no Brasil: análise dos dados da PNAD de 2004. Segurança Alimentar e Nutricional, v. 15, n. 1, p. 49-61, 2008.

IBGE. Microdados da POF 2008-2009 (Pesquisa de Orçamentos Familiares). [2009]. Disponível em: http://www.ibge.gov.br/home/estatistica/populacao/condicaodevida/pof/ 2008_2009/microdados.shtm.

IBGE. Tabela de composição nutricional dos alimentos consumidos no Brasil 2008-2009. Rio de Janeiro, 2011. Disponível em https://biblioteca.ibge.gov.br/visualizacao/livros/liv50002.pdf.

IPEA et al. Retrato das desigualdades de gênero e raça:1995 a 2015. 4. ed. Brasília, 2011. Disponível em: https:/www.ipea.gov.br/retrato/pdf/revista.pdf. Acesso em: 8 dez. 2020.

KEPPLE, A.; SEGALL-CORREA, A. Conceptualizing and measuring food and nutrition security. Ciência E Saúde Coletiva, v. 16, n. 1, p. 187-199, 2011.

OLIVEIRA, J. S. et al. Insegurança alimentar e estado nutricional de crianças de Gameleira, zona da mata do Nordeste brasileiro. Revista Brasileira de Saúde Materno Infantil, v. 10, n. 2, p. 237-245, 2010.

ONU. Fome zero e agricultura sustentável. In: Documentos temáticos: objetivos de desenvolvimento sustentável. Brasília: ONUBR, 2017. p. 14.

PANIGASSI, G. et al. Insegurança alimentar como indicador de iniqüidade: análise de inquérito populacional. Cadernos de Saúde Pública, v. 24, n. 10, p. 2376-2384, 2008.

PIMENTEL, P. G.; SICHIERI, R.; SALLES-COSTA, R. Food insecurity, socioeconomic conditions and anthropometric indicators in children living in the metropolitan region of Rio de Janeiro, Brazil. Revista Brasileira de Estudos de População, v. 26, n. 2, p. 283-294, 2009.

POULAIN, J. P.; PROENÇA, R. P. D. C. O espaço social alimentar: um instrumento para o estudo dos modelos alimentares. Revista de Nutrição, v. 16, n. 3, p. 245-256, 2003. 
SAMPAIO, M. D. F. A. et al. (In) segurança alimentar: experiência de grupos focais com populações rurais do Estado de São Paulo. Segurança Alimentar e Nutricional, v. 13, n. 1, p. 64-77, 2006.

SEGALL-CORREAA, A. M.; MARIN-LEON, L. A segurança alimentar no Brasil: proposição e usos da escala brasileira de medida da insegurança alimentar (EBIA) de 2003 a 2009. Segurança Alimentar e Nutricional, v. 16, n. 2, p. 19, 2009.

SMITH, L. C. The use of household expenditure surveys for the assessment of food insecurity. In: FAO. Measurement and assessment of food deprivation and undernutrition. Rome, 2002.

UNITED NATIONS. The millennium development goals report 2015. [S. 1.]: UN, $2015 \mathrm{a}$.

UNITED NATIONS. General assembly of the United Nations. 2015b. Disponivel em: https:// www.un.org/en/ga/. Acesso em: 9 dez. 2020.

WHO. Guideline: assessing and managing children at primary health-care facilities to prevent overweight and obesity in the context of the double burden of malnutrition. Geneva, 2017.

WHO. Physical status: the use and interpretation of anthropometry. Technical Report Series, n. 854, 1995.

WOOLDRIDGE, J. M. Econometric analysis of cross section and panel data. Cambridge: The MIT Press, 2007.

YUYAMA, L. K. O. et al. Segurança/insegurança alimentar em famílias urbanas e rurais no estado do Amazonas: I. validação de metodologia e de instrumento de coleta de informação. Acta Amazónica, v. 37, n. 2, p. 247-252, 2007.

ZAIDI, M. A.; HAGENAARS, A. J. M.; DE VOS, K. Poverty statistics in the late 1980s: research based on micro-data. Luxembourg: EUROSTAT, 1994.

\section{Autor correspondente:}

Cícero Augusto Silveira Braga

Recebido em: 28/10/2018.

E-mail: ciceroaugustosb@gmail.com

Aceito em: 23/05/2019.

\section{(cc) BY}

